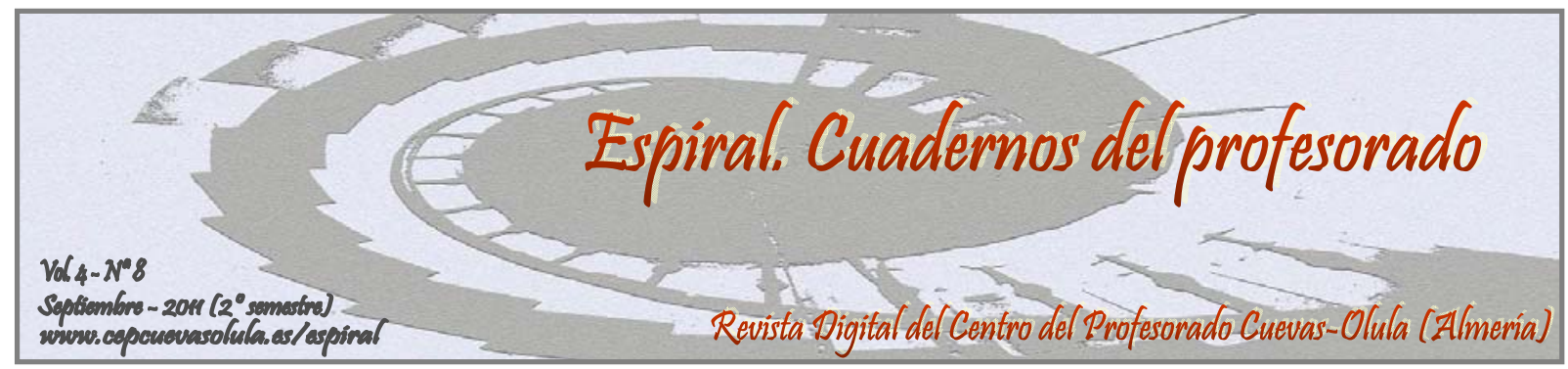

\title{
ESTRATEGIAS Y METODOLOGÍA FRENTE AL DESARROLLO DE UNA ACCIÓN FORMATIVA VIRTUAL: UNA PROPUESTA PRÁCTICA CON ALUMNADO EN EDUCACIÓN SECUNDARIA
}

\section{STRATEGIES AND METHODS IN THE DEVELOPMENT OF VIRTUAL TRAINING ACTION: A PRACTICAL PROPOSAL TO STUDENTS IN SECONDARY EDUCATION}

\author{
María José Ramos Alcázar ${ }^{(1)}$ y Francisco José Martínez López ${ }^{(2)}$
}

${ }^{(1)}$ I.E.S. Pueblos de la Villa, Fuente Álamo, Región de Murcia, España

${ }^{(2)}$ I.E.S. Ricardo Ortega, Fuente Álamo, Región de Murcia, España

RESUMEN: La función tutorial virtual desempeña cada vez un papel más importante en las actuales modalidades educativas online. A través de este trabajo se presenta una propuesta formativa virtual que realza el papel del tutor, a la vez que se establecen unas estrategias metodológicas diseñadas para que el alumnado, no iniciado en este modelo formativo, adquiera unos conocimientos y desarrolle unas competencias de forma autónoma. Para ello se recurre a diferentes modalidades de agrupamientos con el alumnado, junto a una pertinente utilización de recursos TIC, que se complementarán con una intervención planificada del profesor como tutor virtual. De este modo, además de alcanzar los objetivos previstos, se desarrolla la acción formativa en una doble vertiente: los relativos a la especificidad de la materia y los vinculados a adquisición de habilidades y conocimientos propios de esta modalidad formativa.

Palabras clave: tutor online, entorno virtual de aprendizaje, estrategia metodológica, agrupamientos del alumnado, acción formativa virtual.

ABSTRACT: The virtual tutorial role is increasingly playing a larger role in the current online educational methods. Through this work, a virtual training proposal that enhances the role of the tutor, while introducing some methodological strategies designed to make not initiated students in this formative model, acquire knowledge and develop competencies autonomously. This is done by different types of grouping with the students, who will provide, along with a relevant use of ICT resources, development of teaching-learning process successfully through a virtual training activity. Thus, in addition to achieving the objectives, the training takes place in two ways: those relating to the specific matter and those related to acquisition of skills and own knowledge of this training modality.

Key words: online tutor, virtual environment of learning, grouping of students, ICT resources, educational methods. 
Ramos Alcázar, M.J. y Martínez López, F. J. (2011). Estrategias y metodología frente al desarrollo de una acción formativa virtual: una propuesta práctica con alumnado en Educación Secundaria. Espiral. Cuadernos del Profesorado, 4(8), 32-41. Disponible en: http://www.cepcuevasolula.es/espiral.

Fecha de recepción: 13/02/2011

Fecha de aceptación: 12/05/2011
Enviar correspondencia a: mariajoseramosalcazar@gmail.com

\section{1.- INTRODUCCIÓN. LA IMPORTANCIA DE LA TUTORÍA VIRTUAL}

Ante un desarrollo tecnológico, cada vez más avanzado y acelerado como en el que actualmente nos hallamos inmersos, se nos hace más evidente el retraso que supone la educación formal ante la demanda formativa actual, como señala Ortega Sánchez (2007). Actualmente es obvio que el avance tecnológico y el uso de las nuevas tecnologías en la educación ofrecen amplias posibilidades a la formación virtual. En esta línea se incardina esta propuesta práctica, con el fin de ampliar las posibilidades educativas hacia nuestros discentes, en un intento de anticiparnos a una tendencia que se presenta imparable y con un potencial de aprendizaje aún por desarrollar.

Se trata de un intento de acercamiento a una nueva estrategia de enseñanza, la educación virtual tutorizada, propuesta a un alumnado que se introducirá en ella por primera vez. Esta situación conlleva, todo un reto al docente, que deberá doblegar esfuerzos para facilitar el proceso de enseñanza-aprendizaje.

Para ello tendrá que desempeñar sus funciones como tutor virtual, atendiendo convenientemente a los roles, desarrollando las habilidades pertinentes y el conjunto de competencias que las determinan (Mason, 1991; Ryan, Scott, Freeman y Patel, 2000; Cabero, 2004).

El tutor virtual no difiere mucho del tutor presencial, ya que ambos comparten un objetivo común: "ayudar a que los alumnos aprendan"; Martínez (2004) añade al respecto que incluso debe favorecer que las personas aprendan a pensar y decidir por sí mismas. Incluso, considera que los tutores van a tener que especializarse en aquellos aspectos que sean mejores que los ordenadores.

Existen multitud de autores que aportan sus definiciones y consideraciones de esta figura del tutor, pero añadiremos la recogida por Cabero (2011) que lo considera como: "Profesor que además de realizar su labor pedagógica, desempeña tareas relacionadas con el desarrollo personal, académico y social de los alumnos, bien de forma individual o en grupo en función del nivel educativo en el cual se desenvuelva" (p. 18).

Se ha de tener en cuenta que con los nuevos medios y entornos de aprendizaje online que surgen con las nuevas tecnologías, se debe de adoptar nuevas funciones y roles para gestionar esta labor. Si se atiende a Padula (2002), citado por Valverde y Garrido (2005), la función tutorial es entendida como:

"La relación orientadora de uno o varios docentes respecto de cada alumno en orden a la comprensión de los contenidos, la interpretación de las descripciones procedimentales, el momento y la forma adecuados para la realización de trabajos, ejercicios o autoevaluaciones, y en general para la aclaración puntual y personalizada de cualquier tipo de duda" (p. 1).

Pero centrándonos en este contexto particular en que nos hallamos, referido al tutor virtual, se encuentran una multitud de novedades, circunstancias y elementos propios de este entorno, que como consecuencia se derivan en nuevas funciones para el tutor. Muchas de las cuales han sido estudiadas y analizadas por un número no pequeño de autores, en muchos y variados estudios. Es el caso de Ryan, Scott, Freeman y Patel (2000); Paulsen (1995), Mason (1991), Collis y Berge (1995), García Aretio (2006) citados por Cabero (2011), cuyas aportaciones han coincidido en algún caso, o se han 
complementado en otros. Recopilamos aquí las funciones que establece Cabero (2004) y que se establecen como el referente para llevar a cabo el desarrollo de esta propuesta. Éstas son:

1.- Función técnica del tutor.

2.- Función social.

3.- Función académica.

4.- Función orientadora.

5.- Función organizativa.

Por otro lado, en el desarrollo de estas funciones a la hora de realizar las actividades previstas con el alumnado, deberá poseer una serie de habilidades. Berge (1995) recoge algunas como:

- Tener claro los objetivos de participación.

- Mantener un estilo de comunicación no autoritario.

- Animar a la participación.

- Ser objetivo y considerar el tono de intervención.

- Presentar opiniones conflictivas.

- Alabar y reforzar públicamente las conductas positivas.

- No ignorar las conductas negativas, pero llamar la atención de forma privada.

Por otro lado, Duggley (2001) propone las siguientes:

- Saber iniciar y cerrar los debates.

- No creer en que debe ser siempre el tutor el que inicie las participaciones.

- Comenzar cada nuevo debate pidiendo la contribución de un estudiante.

- Intervenir de vez en cuando para hacer una síntesis de las intervenciones.

Sin olvidar las que Salmon (1999) nos aporta:

- Ser amable

- Ser atento, respetuoso y cortés.

- Comprometerse con las personas de una manera no mecánica.

- No escribir demasiado, ser conciso.

- Habilidad para interaccionar y archivar la interacción.

- Tener sensibilidad cultural.

Tabla 1.- Competencias del profesor tutor virtual. Fuente: Barker (2002).

\begin{tabular}{|c|c|c|}
\hline Competencias Pedagógicas & Competencias Técnicas & Competencias Organizativas \\
\hline $\begin{array}{ll}\text { - } & \text { Estructurar el conocimiento. } \\
& \text { Diseño de tareas para el } \\
\text { autoaprendizaje } \\
\text { - } \quad \text { Diseño de actividades en } \\
\text { equipos } \\
\text { - } \quad \text { Formular estrategias de } \\
\text { valoración } \\
\text { - } \quad \text { Guiar, aconsejar y } \\
\text { proporcional feedback. }\end{array}$ & 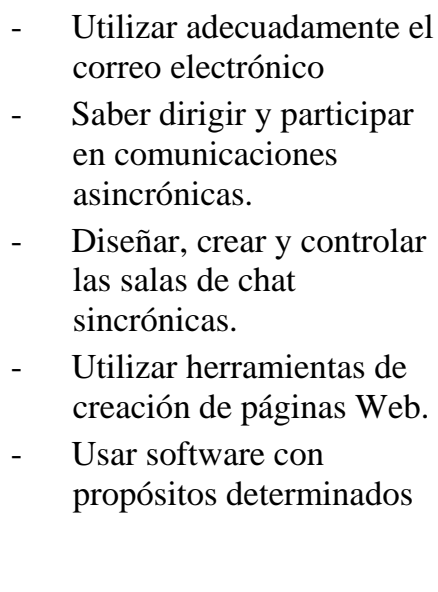 & $\begin{array}{ll}\text { - } & \begin{array}{l}\text { Organización de los } \\
\text { alumnos para realizar tareas } \\
\text { en equipos. }\end{array} \\
\text { - } & \begin{array}{l}\text { Establecer estructuras en la } \\
\text { comunicación on-line con }\end{array} \\
\text { una determinada lógica. } \\
\text { - } & \begin{array}{l}\text { Organizar los estudiantes de } \\
\text { acuerdo a sus datos }\end{array} \\
\text { geográficos. } \\
\text { - }\end{array}$ \\
\hline
\end{tabular}


En definitiva, dentro del proceso formativo en el que nos encontramos, el profesor tutor debe de estar en posesión de un conjunto de competencias, tal y como indica Barker (2002) para desarrollar las actividades y habilidades antes mencionadas (tabla 1).

Teniendo en cuenta lo anterior, a través de esta propuesta práctica se pretende alcanzar los siguientes objetivos:

a) Conocer la importancia de la comunicación entre los agentes intervinientes en una acción formativa virtual.

b) Valorar la propuesta práctica en cuanto al aprendizaje de los contenidos específicos trabajados por el alumnado participante.

c) Adquirir estrategias metodológicas para diseñar procesos de aprendizaje a través de un planteamiento educativo no presencial en alumnado no habituado a esta modalidad formativa.

d) Proponer una planificación que contribuya al desarrollo de la actividad formativa de manera clara y efectiva

e) Reconocer la importancia de la figura del profesor como tutor virtual en este tipo de propuestas educativas online.

\section{2.- LA ACCIÓN FORMATIVA EN EL AULA VIRTUAL. DISEÑO Y PLANIFICACIÓN DE LA PROPUESTA}

La acción formativa virtual a llevar a cabo consiste en el desarrollo de una unidad didáctica en inglés donde el alumnado deberá desarrollar las destrezas de comunicación escrita (writing) con sus competencias, a través de unos temas propuestos por el profesor.

El grupo de alumnado al que va destinada esta acción formativa es de 60 estudiantes en total, de bachillerato. Se trata de un grupo bastante heterogéneo ya que proceden en igual número del bachillerato de las modalidades de Ciencias y Tecnología, de Humanidades y Ciencias Sociales y de Artes. Esta característica nos va servir para que enfoquemos las tareas a desarrollar, de la forma más productiva y enriquecedora posible por las aportaciones que van a ir realizando los alumnos y alumnas desde su itinerario académico. Además, tomaremos dichos perfiles para establecer las estrategias didácticas que se van a poner en práctica, ya que van a corresponder a tipos de trabajos colaborativos: donde se irán arbitrando distintas opciones que el mismo ofrece, atendiendo a la organización en grupos para la realización de este trabajo.

Tabla 2.- Propuesta de planning para la actividad "Problems in 21st Century”. Fuente: Elaboración propia.

\begin{tabular}{ll}
\hline CALENDAR & \multicolumn{1}{c}{ TASKS } \\
\hline PART 1: June 1-3 & -Study the materials in the unit “TODAY'S PROBLEMS”. \\
\hline & -Wiki training in Group Work Space Wiki \\
\hline PART 2:June 6 - 10 & -Brainstorming (in writing) in the Group Work Space Wiki. \\
& -Write an individual essay and send it to your Group work Space Wiki. \\
& -Read and correct each other's individual essays in group wiki. \\
\hline & -Revise your own individual essay in your group wiki. \\
PART 3:June 13-16 & -Prepare collaborative group essay by editing and adding an \\
& introduction and conclusion. \\
\hline & -Post finished essay to forum for the class to read. \\
\hline
\end{tabular}


Atendiendo a Barberá (2008) en cuanto al diseño de una acción formativa en un aula virtual, como es el caso, se ofrece el siguiente planteamiento para el desarrollo de la misma.

OBJECTIVES:

- Read articles about recent problems in the world.

- Formal writing on opinions stated by others.

- $\quad$ Practice using connectors.

- Revising your own writing.

- Collaborative writing

- $\quad$ Providing your team-mates with feedback on their writing.

\section{CONTENTS:}

\section{- TOPICS:}

\section{NUCLEAR ENERGY.}

2. SPANISH REVOLUTION. MAY 15th

\section{MOVIES AND INTERNET}

\section{GM FOOD: THE ANSWER TO THE HUNGER IN THE WORLD.}

- GRAMMAR: Present, past tenses, Connectors (...)

RESOURCES: Para desarrollar este planning de trabajo (tabla 2) por vía telemática, lo haremos a través de la plataforma de formación virtual moodle, que dispone el centro de bachillerato al que nos referimos. Concretamente, precisaremos del foro, correo electrónico, wiki, chat..., que nos ofrece este sistema. Además de las tareas específicas que se han descrito que estarán allí alojadas.

EVALUATION:

- Individual mark:

20\% Participation in Group Work Space

$50 \%$ Individual essay added to group wiki

- Group mark:

$30 \%$ Final document in group wiki (group revision)

Tras la presentación de esta planificación, o diseño, se atiende a los siguientes módulos: módulo guía (se identificaría con los objetivos propuestos) módulo de contenidos (los contenidos), módulo de comunicación (herramientas presentadas) y el módulo de evaluación (en este caso, se especifica los criterios de calificación que los estudiantes deben conocer).

Para alcanzar un funcionamiento óptimo de la acción formativa virtual planificada, el profesor tutor (que ya se habrá identificado y que para este caso, se trata de un profesor de inglés), tomará como referente los cinco pasos que propone Salmon (2004).

Por tanto, en el primer paso, dicho profesor iniciará el proceso dando las instrucciones precisas y necesarias a los alumnos y alumnas que configuran este grupo para que accedan al sistema, a la vez que les irá dando la bienvenida al mismo, animándoles para que vayan consiguiendo la confianza necesaria para empezar a desarrollar las primeras tareas. El inicio irá desarrollándose según lo establecido en la parte $1^{\text {a }}$ del planning (tabla 2).

En el segundo paso, se inicia la actividad propiamente en una fase de iniciación, donde a la vez que van trabajando las tareas propuestas, se trata de conseguir la cohesión del grupo procurando desarrollar un camino sistemático de trabajo on-line, a la vez que se cuida el grado de socialización correcto en línea tanto con el intercambio de información que realicen, como con el tipo de comunicación que desarrollen. Ya nos podemos situar en la parte 2. 
En el tercer paso, hemos de procurar que los estudiantes se vayan involucrando cada vez más en cuanto que se ofrezcan, reconozcan diferentes estilos de aprendizaje y organicen la información que van a construir. Comienzan con el verdadero proceso de aprendizaje, ya que interaccionan con los materiales (sus propios textos, desde sus propias correcciones) con sus compañeros y con el profesor tutor (cuando éste les ha corregido y devuelto los trabajos para que continúen el proceso).

Continuamos con el cuarto paso, donde vamos dejando a los estudiantes que vayan trabajando entre ellos. En estos momentos la figura del profesor tutor va pasando a un segundo plano. De este modo, se intenta que vayan construyendo su propio conocimiento a partir de las correcciones recibidas del trabajo colaborativo que deben ir realizando, al afrontar y resolver los conflictos que les ocurra. Estamos hablando de la parte $3^{\text {a }}$ del planning.

Finalmente, el quinto paso coincide con la parte $4^{\mathrm{a}}$ programada, donde ya los estudiantes van alcanzando mayor grado de independencia. La responsabilidad que van adquiriendo ellos mismos en su propio aprendizaje, la van a ir adquiriendo del resto de trabajos aportados por sus compañeros, a la vez que van concluyendo este proceso de formación virtual.

\section{3.- RESULTADOS Y DISCUSIÓN}

Tomando como punto de referencia el planning (tabla 1) se puede detectar cuáles son las técnicas de trabajo que se pretenden desarrollar en los estudiantes. Resaltar, por un lado la parte $1^{\mathrm{a}}$ en la que la individualización de la enseñanza es más clara y oportuna, pues el estudiante debe recuperar información y estudiar los materiales interactivos; Continuamos con el trabajo colaborativo, abordando en éste ultimo desde la lluvia de ideas hasta, estudios de casos, método de proyectos, wikis...

Dada la composición del grupo sobre el que se plantea la propuesta a desarrollar, se pasa a detallar la organización concreta de la misma para que como resultado final se obtenga el enriquecimiento mutuo. Se pretende que tras la culminación del proceso formativo todos aprendan de todos.

En este sentido y respondiendo a la función organizativa del tutor virtual, según Cabero (2004), se realiza la organización del trabajo en equipos, facilitando la coordinación entre sus miembros, de este modo se atiende a un trabajo colaborativo, posibilitando la atención a la riqueza diversa que ocupa nuestras aulas, de manera más centrada y próxima a sus verdaderos intereses y favoreciendo el desarrollo de la identidad personal en condiciones de equidad y calidad (Ramos Alcázar y Martínez López, 2010).

Las fases organizativas son las siguientes:

a) FASE 1: FORMACIÓN DE EQUIPOS EN CADA UNA DE LAS CLASES.

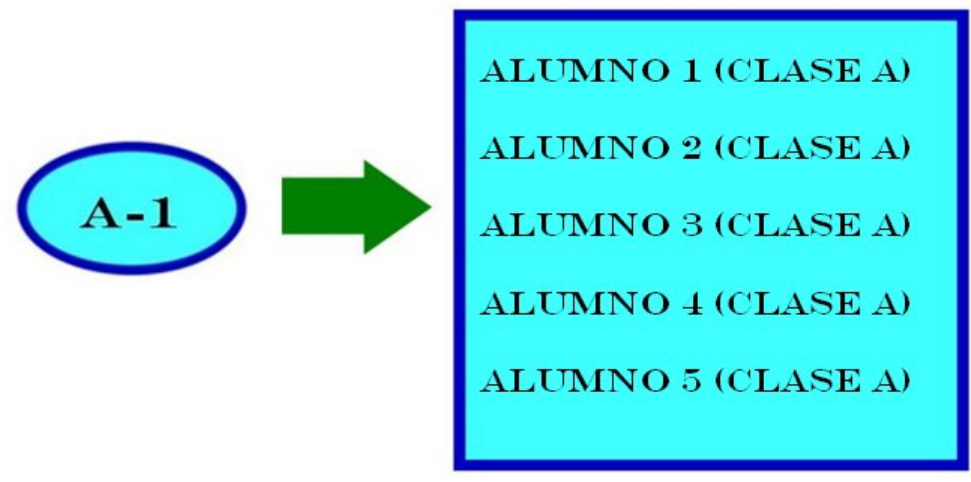

Figura 1.- Formación del equipo A-1 para llevar a cabo una formación educativa virtual. Fuente: Elaboración propia.

Atendiendo a una de las funciones sociales del tutor virtual, tal y como recoge Cabero (2004), se ha de facilitar la creación de equipos de trabajo. En este sentido se formarán agrupamientos de 5 alumnos/as entre los existentes en cada de una de las clases que intervienen en la formación virtual. De 
este modo en la clase A tendremos 4 grupos de 5 personas cada uno. Estos grupos pasarán a denominarse equipos A-1, A-2, A-3, A-4 y A-5, para la clase A. Así, por ejemplo, el equipo A-2, estará formado por los alumnos 6, 7, 8, 9 y 10, el equipo A-3 por los alumnos 11, 12, 13, 14 y 15, así sucesivamente. La decisión e iniciativa para la formación de los equipos será de los propios alumnos y alumnas. El profesor sólo intervendrá en el caso de que existan dificultades para los agrupamientos.

Igualmente, en las clases B y C habrá sucedido lo mismo, teniendo, por tanto, los equipos B-1, B-2, B3, B-4, B-5, C-1, C-2, C-3, C-4 y C-5.

\section{b) FASE 2: RELACIÓN ENTRE LOS EQUIPOS DE LAS DIFERENTES CLASES.}

En esta fase se establecerá una actividad conjunta entre equipos, concretamente entre un equipo de cada una de las 3 clases. Así, por ejemplo, el equipo A-1, durante esta fase de la formación educativa virtual, desarrollará la actividad con el equipo B-1 y el C-1. De este modo, también tendremos que el equipo A-2 se relacionará con el B-2 y C-2. Así sucesivamente, tal y como dejo reflejado en la figura 2:

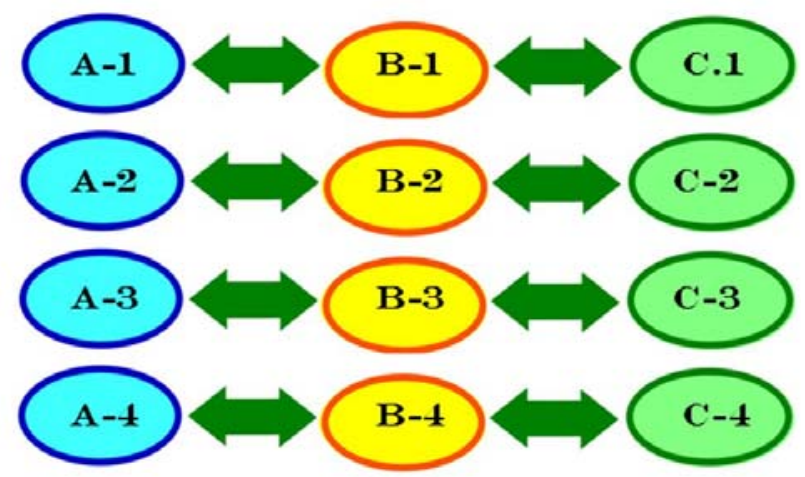

Figura 2.- Relación entre los equipos de las 3 clases inmersas en la experiencia de formación educativa virtual. Fuente: Elaboración propia.

\section{c) FASE 3: PUESTA EN COMÚN DEL TRABAJO FINAL.}

Cuando cada uno de los grupos haya abordado sus temas sujetos a estudio, desde su punto de vista (científico con los equipos A, social con los equipos B y político con los equipos C), se obtendrá un único trabajo con la conjunción de los 3 puntos de vista. Esto se repetirá para cada uno de los 4 temas que fueron propuestos por el/la profesor/a. De este modo, se obtendrán 4 trabajos con 3 puntos de vista cada uno que serán llevados al foro. Esquemáticamente, y para los equipos A-1, A-2 y A-3, quedaría como se muestra en la figura 3:

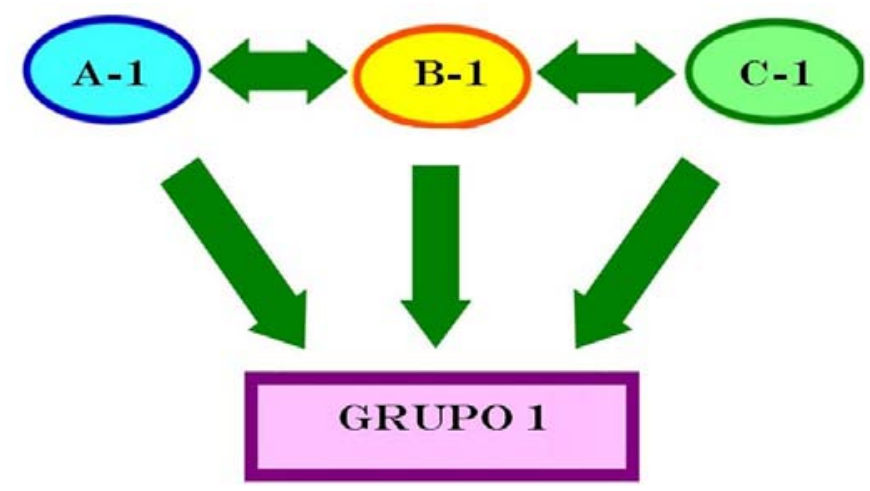

Figura 3.- Puesta en común en un único trabajo (el correspondiente al GRUPO 1) los 3 puntos de vista de los equipos A-1, B-1 y C-1. Fuente: Elaboración propia. 


\section{d) FASE 4.- PUESTA EN COMÚN AL GRAN GRUPO.}

Los trabajos de todos los grupos son expuestos en el foro general (figura4), de manera que todos los alumnos y alumnas de todas las clases acceden a la totalidad de los trabajos desarrollados.

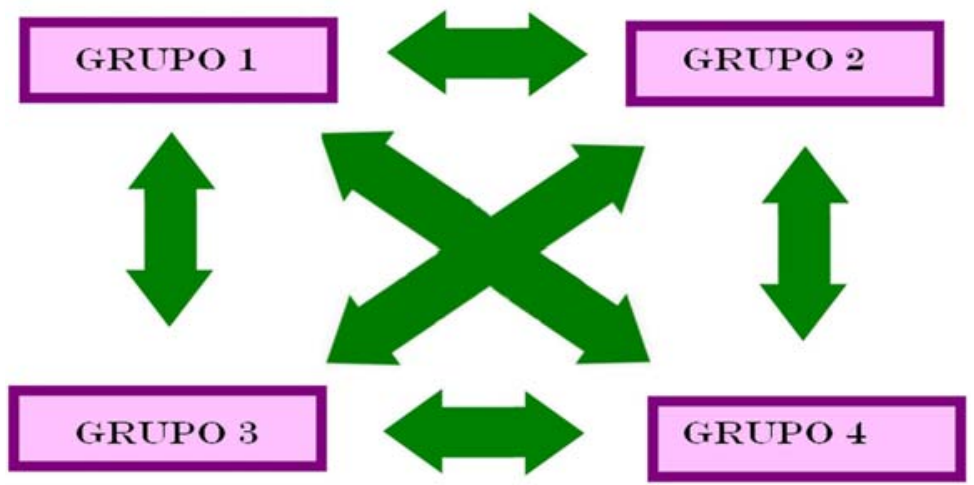

Figura 4.- Puesta en común de todos los trabajos por parte de todos los grupos. Fuente. Elaboración propia.

\section{4.- REPERCUSIONES EN LA COMUNIDAD EDUCATIVA}

Los entornos no presenciales de aprendizaje permiten abordar la formación de tres dimensiones básicas: la adquisición de habilidades cognitivas para el manejo de información multimedia, el conocimiento y manejo de herramientas y aplicaciones informáticas; y el desarrollo de una actitud crítica y reflexiva para valorar tanto la información, como las medios tecnológicos disponibles.

A través de esta propuesta práctica se plantea una estrategia metodológica enfocada a un alumnado que se presupone no iniciado en la modalidad formativa virtual. Esta circunstancia conlleva tener presente la necesidad de incidir en aquellos aspectos que pueden intervenir negativamente en el proceso pudiéndolo hacer fracasar al no permitir la consecución de los objetivos previstos. En este sentido, se considera que la falta de habilidades TIC en el alumnado pueden representar un hándicap para el desarrollo satisfactorio de la experiencia propuesta.

También es importante el grado de implicación del profesorado, pudiendo representar un elemento determinante. El docente debe disponer de una óptima predisposición a este tipo de experiencias. Además, los docentes como protagonistas fundamentales de la innovación educativa que se pretende implementar, deberían estar sujetos a políticas de apoyo institucionales (Salinas, 2010). Es primordial que los docentes conozcan las funcionalidades técnicas y las potencialidades didácticas de los entornos virtuales, como paso previo para su integración significativa en las propuestas curriculares.

Sin duda, las TIC, representan un valioso recurso de apoyo a las actividades docentes, aunque la solución no está en los teclados, ni aún en la red, sino en la motivación docente y en la formulación de políticas educativas integrales que atiendan las necesidades de la educación para el siglo XXI (Delors, 1996).

Superado este escollo, el planteamiento de propuestas educativas no presenciales en alumnado de Educación Secundaria representa una innovación educativa tecnológica. Aunque aún no está asentada, irá adquiriendo una mayor presencia, anticipando y, sobre todo, preparando al alumnado a asimilar nuevos modelos educativos en niveles superiores.

\section{5.- CONCLUSIONES}

En primer lugar, es necesario destacar que la comunicación entre el alumnado y el profesor tutor tiene una enorme importancia en este proceso educativo, también entre el propio alumnado participante. El diálogo, el intercambio que nos hace reflexionar acerca de lo que los otros nos aportan, compararlo con nuestra propia opinión y construir una respuesta constituye un proceso formativo verdadero. No aprenderemos mucho de aquellos que piensan igual que nosotros. La diversidad de conocimientos e ideas suponen un enriquecimiento formativo y educativo. 
En segundo lugar, teniendo en cuenta el contenido temático que se ha propuesto para trabajar en esta acción formativa virtual, el aprendizaje se nos presenta verdaderamente significativo desde una doble vertiente: por un lado, en la específica que conlleva la enseñanza del aprendizaje propio de la lengua extranjera (contenido de aprendizaje: vocabulario de los temas sugeridos en inglés, aspectos gramaticales, funciones lingüísticas empleadas, etc.) que desarrollará las competencias comunicativas mediante la práctica real, contextualizada, con una intención comunicativa real. Por otro lado, y en paralelo con lo anterior, el aprendizaje efectivo con el manejo y puesta en práctica de las estrategias y recursos online presentados a los largo del desarrollo de esta acción formativa virtual. Sendos aprendizajes, se adquirirán con verdadera efectividad y significatividad, ya que estos estudiantes no recibirán una simple exposición y trasmisión de información, datos y teoría para adquirir los conocimientos previstos, sino que mediante el desarrollo y resolución de unas tareas expuestas, serán conducidos finalmente a lograr unos objetivos previstos.

Además, no nos podemos olvidar de la importancia que adquiere en una propuesta formativa de estas características una planificación comprensible, precisa y adaptada plenamente a las intencionalidades de la estrategia didáctica elegida, la de una actividad formativa virtual.

Finalmente, hemos de remarcar la importancia de la figura del tutor virtual, convirtiéndose en una pieza fundamental, representando un papel clave para el eficaz desarrollo del trabajo colaborativo propuesto.

\section{6.- REFERENCIAS BIBLIOGRÁFICAS}

Barberá, E. (2008). Aprender e-learning. Barcelona: Paidós.

Barker, P. (2002): Skill sets for online teaching, en Ed-Media, World Conference on Educational Multimedia, Hypermedia and Telecomunications.

Berge, Z. (1995). Facilitating computer conferencing: recomendations from the field, Educational Technology $35(1) 22-30$.

Cabero, J. (2004). La función tutorial en la teleformación, en F. Martínez y M. P. Prendes (coords.): Nuevas tecnologías y Educación. Madrid: Pearson.

Cabero (2011). Modalidades de acción tutorial. En UDIMA, Nuevos modelos y recursos en la práctica docente. Madrid.

Collis, M.P. y Berge, Z. (1995). Moderating online discussion groups. Disponible en: http://www.emoderators.com/moderators/sur_aera97.html [Consulta: 2011, 28 de enero].

Delors, J. (1996). La educación encierra un tesoro. En J. Delors, La educación encierra un tesoro. Madrid: Santillana.

Duggley, J. (2001). El tutor on line. La enseñanza a través de internet. Bilbao: Deusto.

García Aretio, L. (2006) (coord.). De la educación a distancia a la educación virtual. Barcelona: Ariel.

Martínez, J. (2004). El papel del tutor en el aprendizaje virtual [en línea]. UOC. Disponible en: http://www.uoc.edu/dt/20383/index.html [Consulta: 2011, 7 de enero].

Mason, R. (1991). Moderating educationalcomputer conference. Desosnews, 1, 19.

Ortega Sánchez, I. (2007). El tutor virtual: aportaciones a los nuevos entornos de aprendizaje. Revista Electrónica Teoría de la Educación. Educación y Cultura en la Sociedad de la Información, 8(2), 100115.

Padula, J.E. (2002) Contigo en la distancia. El Rol del tutor en la Educación No Presencial. Disponible en: http://www.uned.es/catedraunesco-ead/publicued/pbc08/rol_bened.htm [Consulta: 2011, 2 de febrero].

Paulsen, M. (1995). Moderating educational computer conferences. Disponible en: http://www.nettskolen.com/alle/forskning/20/moderating.html [Consulta: 2011, 12 de enero].

Ramos Alcázar, M. J. y Martínez López, F. J. (2010). El agrupamiento flexible: un marco organizativo como medio para la atención a la diversidad en la Educación Secundaria Obligatoria [en línea]. Espiral. Cuadernos del Profesorado, 3(6), 65-72. Disponible en: http://www.cepcuevasolula.es/espiral [Consulta: 2010, 29 de diciembre].

Ryan, S., Scott, B., Freeman, H. y Patel, D., (2000). The virtual university, the Internet and resource-bases learning. London: Kogan Page. 
Salinas, M.I. (2010). Diseño de políticas docentes para la adopción de la enseñanza virtual: el caso de un departamento universitario. Revista Electrónica de Tecnología Educativa 33, 1-16. Disponible en: http://edutec.rediris.es/Revelec2/Revelec33/pdf/Edutec-e_n33_Salinas.pdf [Consulta: 2011, 4 de febrero].

Salmon, G. (1999). Reclaiming the territory for the natives. Disponible en: http://www.emoderators.com/moderators/gilly/LONDON99.HTML [Consulta: 2011, 20 de enero].

Salmon, G. (2004). E-actividades. El factor clave para una formación en línea activa. Barcelona: UOC.

Valverde, J. y Garrido, M. C. (2005). La función tutorial en entornos virtuales de aprendizaje: comunicación y comunidad, Revista Latinoamericana de Tecnología Educativa 4 (1). Disponible en: http://campusvirtual.unex.es/revistas/index.php?journal=relatec\&page=article\&op=view\&path\%5B\%5 $\underline{\mathrm{D}=195}$ [Consulta: 2011, 28 de enero].

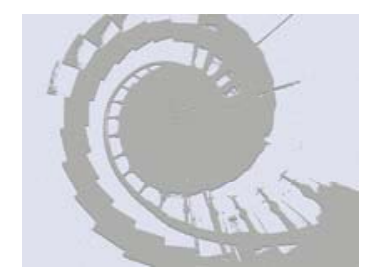

